Ogueji, A. Ifeanyichukwu, ${ }^{1}$ Okoloba, M. Maia. ${ }^{2}$

\title{
Compassion-Focused Therapy (Cft) As An Intervention Against Suicidal Ideation In Newly Diagnosed People Living With Hiv/Aids (Plwha) Attending A Nigerian Maternity Teaching Hospital
}

\author{
'Department of Psychology, University of Ibadan, Ibadan, Nigeria \\ 2Department of Psychology, The University of Buckingham; and Lewisham and Greenwich NHS Trust, United Kingdom \\ *email: ifeanyiogueji21@gmail.com
}

DOI: 10.2478/gp-2020-0012

Received: 4 April 2020; Accepted: 22 April 2020

\begin{abstract}
Objectives: In Sub-Saharan Africa, Nigeria is currently home to many people living with HIV/AIDS (PLWHA), and suicidal ideation is amongst the common early problems of newly diagnosed PLWHA. This reflects the importance of including psychotherapy in HIV care. In this regard, compassion-focused therapy (CFT) is the one that has not been a point of study for reducing suicidal risks in newly diagnosed PLWHA despite the relevance of CFT to the treatment/management of persons living with self-criticism or self-harm. To bridge this gap, we examined the use of CFT as an intervention against suicidal ideation in newly diagnosed PLWHA receiving antiretroviral therapy at the Adeoyo Maternity Teaching Hospital in Ibadan, Oyo state, Nigeria.

Methods: Twenty-two participants (mean age $=40 \pm 5.6$ years) with less than 1-month-old clinical diagnosis of HIV who have recently thought about suicide because of the new HIV-positive diagnosis were purposively sampled and randomly assigned into the intervention and control groups. The CFT module and scale for suicide ideation were the research instruments. All data collected were analysed using SPSS (v.22.0), and statistical significance was determined at $P<0.05$.

Results: The findings established that after administering CFT to the intervention group, the mean score for suicidal ideation was significantly lower in the intervention group compared with that in the control group.

Conclusion: The results suggest that CFT has the potential to reduce suicidal ideation in newly diagnosed PLWHA; therefore, in addition to the antiretroviral therapy, the administration of CFT should be included in the early stage of HIV care, to reduce the risk of suicide that is associated with newly living with HIV/AIDS.
\end{abstract}

\section{Keywords}

Compassion-focused therapy (CFT), Suicidal ideation, Newly diagnosed PLWHA, HIV/AIDS.

\section{INTRODUCTION}

HIV/AIDS remains amongst the most dreadful illness affecting the physical and mental health of persons in Sub-Saharan Africa. Related studies have often shown that there are varied psychosocial reactions by newly diagnosed people living with HIV/AIDS (PLWHA), and these reactions may range from fear of impending death to fear of discrimination and suicidal thoughts (Odiachi, et al. 2018; Okoror, Falade, Walker, Olorunlana \& Anaele, 2016; Dabaghzadeh, Jabbari, Khalili \& Abbasian, 2015). Recent statistics showed that Sub-Saharan Africa reports amongst the highest number of cases of HIV/ AIDS amongst adolescents and adults. In addition, HIV/AIDS has been identified as the leading cause of disease burden in Sub-Saharan Africa (Dwyer-Lindgren, et al. 2019). It has been estimated that 6,000 new infections occur globally each day, of which 2 of 3 are in Sub-Saharan Africa, with mostly young women affected (Kharsany \& Karim, 2016). Nigeria accounts for amongst the highest HIV epidemic in the world, one of the highest rates of new infections in Sub-Saharan Africa and one of the highest rates of HIV-related mortality globally. Also, six states in Nigeria have been identified to account for $41 \%$ of PLWHA; they include Kaduna, Akwa Ibom, Benue, Lagos, Oyo and Kano states. The major high-risk groups for HIV infection in Nigeria are sex workers, men who have sex with men and people who inject drugs (Avert, 2019; UNAIDS Data, 2019).

In 2007, Nigeria developed its first HIV prevention plan, guided by the abstinence, be faithful and condom use (ABC) model. In the present time, the major HIV prevention strategy adopted in Nigeria is the revised national HIV and AIDS strategic framework, which is expected to run from 2019 to 2021 (NACA Fact Sheet, 2016; UNAIDS Data, 2019). The new prevention strategy presently implemented in Nigeria is because of the 
increase in the prevalence of HIV/AIDS in the country, which suggests that previous prevention programmes that have been implemented in Nigeria and other parts of Sub-Saharan Africa have not been as effective as envisioned. Therefore, the failure of previous Nigerian HIV prevention programmes could possibly suggest the reason for Nigeria reporting amongst the highest rates of new HIV infection cases and HIV-related mortality in Sub-Saharan Africa. Furthermore, it suggests that there is a need for improved health interventions for PLWHA, especially the newly diagnosed who often present negative psychosocial reactions (such as suicidal tendencies) to a new diagnosis of HIV, because of probable stigma or discrimination that is associated with living with HIV. As a result, this study examined the efficacy compassion-focused therapy (CFT) in reducing suicidal ideations that are observed in newly diagnosed PLWHA in Oyo state, Nigeria, that is amongst the leading states in the prevalence of HIV in Nigeria. The number of studies that have studied how efficacious CFT is on PLWHA in Nigeria does not exist (if any). Most health intervention studies for PLWHA have focused on psychotherapies other than compassion focused therapy, and this creates a knowledge gap on the relevance of CFT as an efficacious health intervention in the management of PLWHA (e.g. Nwobi, et al. 2018). Furthermore, Nigerian studies that have adopted CFT as a health intervention for vulnerable populations were not studied using samples of patients with HIV/AIDS; consequently, findings from such studies are not suitable for generalising to PLWHA (e.g. Agberotimi, Osinowo \& Asagba, 2017).

CFT is a psychotherapy that aims to use compassion as an effective strategy for enhancing well-being or normal functioning in clients. The hallmark of this therapy is that when clients express compassion in their attention, thoughts and behaviours, it reduces their negative responses to negative situations (Gilbert, 2010). CFT acknowledges that pain (physical or emotional pain) is often a part of human experiences and the experience of pains could trigger thoughts of self-harm, self-criticism/condemnation, guilt or shame in humans. Consequently, the CFT works by aiming to modify or change self-harm/suicide or self-criticism to compassionate self-correction through various means of compassionate exercises such as self-regulatory practices, mindfulness and compassionate imagery. This suggests that an imbalance in emotions breeds abnormal thoughts and behaviours; however, the introduction of compassion functions to balance these emotions and enhance normal functioning. Compared to other psychotherapies, the CFT saves clinicians cost to administer and it often yields positive treatment outcomes when used to treat patients with concerns of anxiety, shame, self-criticism, self-injury/suicide or depression (Gilbert, 2014).
Therefore, the current study's premise for adopting CFT as the health intervention for the client group of newly diagnosed PLWHA is that it is expected that if this client group can be trained to be compassionate (compassionate mind training), it would reduce the risk of suicide and self-criticism, which are common problems in them, and the ability of CFT to do this is a major strength of CFT over other psychotherapies such as the acceptance and commitment therapy or cognitive behavioural therapy. The history of CFT can be traced back to the early 21st century, and Paul Gilbert is credited for the development of this therapy. Our review of literature in the next section supports our argument that newly diagnosed PLWHA are often suicidal and self-condemning after receiving a new HIV-positive diagnosis, and this is often triggered from the pain and stigma that they anticipate from living with HIV/AIDS. This, therefore, makes this population potentially able to benefit from CFT.

\section{LITERATURE REVIEW}

\section{Newly Diagnosed PLWHA and Suicidal Ideation}

Agarwal, Mahore and Pathak (2018) established in a sample of patients with less than 1-month-old HIV-positive diagnosis that elements of suicidal ideation, for example, loss of interest in life and depression, were observed as a part of their early reactions to the new HIV-positive diagnosis. Surprisingly, it was found that higher-educated participants reported less negative reactions compared to less-educated participants. Gebremariam, Reta, Nasir and Amdie (2017) in a sample of PLWHA in Addis Ababa, Ethiopia, also established that suicidal ideation and attempt was a common early problem amongst study participants after a new HIV-positive diagnosis. Interestingly, depression, family history of suicide and perceived stigma from living with HIV were found to be associated with suicidal risks in that same study. In a different study, Ruffieux et al. (2019) found that high mortality rate because of suicide is often reported amongst PLWHA, especially the very recently diagnosed (HIV-positive diagnosis less than 1 month old) when compared to the general population. Zarei and Joulaei (2018) identified that suicidal ideation was common in both recently and non-recently diagnosed patients with HIV/ AIDS. Schlebusch and Govender (2015) found that, globally, suicide and HIV/AIDS are two major health issues of concern. Furthermore, they determined that the sample of PLWHA they studied showed an elevated risk of suicide because of the high prevalence of suicidal ideation observed in study participants.

Carrieri et al. (2017) reported from a sample of PLWHA with a diagnosis of less or more than 1 month that high suicidal risks was common after an HIV-positive diagnosis. Lingen-Stallard, 
Furber and Lavender (2016) established in a sample of 13 newly diagnosed HIV-positive women in England that suicidal thoughts, self-condemnation, shock and disbelief, anger and turmoil and confidentiality issues were very common problems in less than 4 weeks after the new HIV-positive diagnosis. Moradmand-Badie et al. (2014) found in a qualitative study that shock, self-harm/suicide, disorientation and self-stigma were commonly observed to be problems amongst patients with HIV/AIDS who were diagnosed in not more than the past 2 weeks. However, the finding further interestingly showed that respondents that were spiritual were found to present less negative reactions and found meaning in life after the new HIVpositive diagnosis. The authors therefore suggested that their study highlights the relevance of pre-HIV testing counselling.

Ongeri et al. (2018) determined in a sample of 394 newly diagnosed Kenyan PLWHA that suicidal ideation was very common amongst participants and that over time suicidal ideation worsened to suicidal attempt amongst $18 \%$ of the study participants. The results are also same in Nigeria, for instance, Onyebueke and Okwaraji (2015) found that the prevalence of suicidal risk was high in newly and previously diagnosed patients with HIV/AIDS attending the University of Nigeria teaching hospital, Enugu state, Nigeria. Similarly, Egbe et al. (2017) found that in a sample of 1187 PLWHA attending 3 HIV treatment centres in Abuja FCT Nigeria, there was a problem of suicide common amongst them. Also, Oladeji et al. (2015) found in a sample of patients with HIV-positive diagnosis that was less than or greater than a month old in Ibadan, Oyo state, Nigeria, that suicidal ideation was the commonest suicidal behaviour amongst participants. Overall, these findings suggest that suicidal ideation is a common early problem in newly diagnosed PLWHA globally, and there is the need for early psychological health intervention with respect to this common early problem, in order to compliment the usual antiretroviral therapy received by this vulnerable group.

\section{CFT and Newly Diagnosed PLWHA}

Although no published study was found to have established the effect of CFT on suicidal risks amongst newly diagnosed PLWHA, the closest to the point of interests of the present study were thus presented. For instance, O'Donovan, Gill and Gibson (2015) found that CFT was found to be useful and supportive in reducing psychological distress in a sample of PLWHA. It was also found that resilience and acceptance of HIV-positive status were enhanced after the exposure to CFT. In a different study, Skinta, Williams and Fekete (2019) determined that compassion-focused practices were significant in reducing HIV stigma and negative affect in PLWHA. Brion,
Leary and Drabkin, (2014) found in a sample of 187 PLWHA that self-compassion was related to better adjustment, lower stress, anxiety, shame and more adaptive reactions to have HIV.

Swansick (2019) in a study that tested self-compassion and shame on a HIV nightline established that self-compassion was imperative for reducing guilt and shame. Harrowing (2011) determined that compassion practice in HIV care significantly fostered well-being amongst PLWHA in Uganda. Surprisingly, in a different study, the experience of HIV symptoms was found to predict reactions to living with HIV amongst PLWHA, although self-compassion was not found to significantly contribute to the experience of HIV symptom in that study (Webel et al. 2015). Differently, Ironson, Kremer and Lucette (2017) established in a longitudinal study that being compassionate towards oneself and others have survival benefits for PLWHA. These findings imply that CFT may have potential benefits for reducing the risks of suicide in newly diagnosed PLWHA, although no published study was found to have demonstrated this. Therefore, this suggests the need to present an empirical study in this regard.

\section{Research Gap}

The elevated risk of suicide is a very common problem in the general population of PLWHA, especially those with less than 1-month-old clinical diagnosis of HIV/AIDS, as demonstrated in literature. CFT is a very important treatment tool over other psychotherapies that could be very useful to the HIVcare multidisciplinary team for reducing suicidal ideation in newly diagnosed PLWHA. Despite the importance of CFT, the number of studies that have shown the efficacy of CFT for reducing suicidal ideation in newly diagnosed PLWHA was scarce, especially in Nigeria where the prevalence of HIV is high.

There are very limited studies that have studied close interests to that of the current study, and a major criticism of such studies was that they reflected the impacts of selfcompassion on different dimensions of health and wellbeing amongst PLWHA, without a focus on the efficacy of CFT in reducing suicidal risks in PLWHA. Hence, findings from such studies cannot be applied to explain the utility of CFT as a health intervention for mitigating suicidal ideation in newly diagnosed PLWHA. To bridge the knowledge gap on the efficacy of CFT in reducing suicidal ideation in newly diagnosed PLWHA (HIV-Positive diagnosis less than a month old), the following research objectives, questions and hypothesis were presented. 


\section{Aim of Study}

The aim was to determine the efficacy of CFT in reducing suicidal ideation amongst newly diagnosed PLWHA.

\section{Research Question}

To what extent will CFT be an efficacious intervention for reducing suicidal ideation in newly diagnosed PLWHA?

\section{Hypothesis}

$\mathbf{H}_{\mathrm{i}}$ Participants exposed to CFT will have significantly reduced suicidal ideation at post-test than at pre-test compared to the participants in the control group.

$\mathbf{H}_{\mathrm{o}}$ There will be no significant difference in suicidal ideation between the intervention and control groups before and after exposure to CFT.

\section{METHODS}

\section{Design}

This study was a randomised controlled trial that adopted a pretest post-test control group design. The rationale for adopting this design was to enable the researcher to assess participants before and after treatment and allow comparisons between the intervention and control groups, to ascertain the efficacy of CFT in reducing suicidal ideation in newly diagnosed PLWHA. The CFT was the independent variable, whereas suicidal ideation was the dependent variable. The Adeoyo Maternity Teaching Hospital, Yemetu, Ibadan, established in 1928 served as the study setting for this study. The high number of new HIV-positive diagnosis that is recorded in the Adeoyo Maternity Teaching Hospital justified the choice for selecting this hospital as the study setting. The hospital is located in Oyo state, Nigeria, and Oyo state is amongst the states accounting for $41 \%$ of the prevalence of HIV/AIDS in Nigeria (Avert, 2019; UNAIDS Data, 2019).

\section{Participants}

The minimum sample size was calculated at $10 \%$ of the total number of patients newly diagnosed with HIV/AIDS at the study setting. Therefore, a total of 22 participants were purposively selected and randomly assigned to the intervention and control groups (11 participants per group). The mean age of total study participants was $40 \pm 5.6$ years. In most studies similar to the current one, experts have always warned of participants' age differences as being a major confounding factor (Kamangar, 2012; Mousa, 2014). Also, there are related studies in which the age differences of participants have been compared between the intervention and control groups to establish its confounding potentiality, and such studies yielded better outcomes (Alavi, Sharifi, Ghanizadeh \& Dehbozorgi, 2013). Therefore, a comparison of age differences between the intervention and control groups was presented in Table 2, to establish the confounding effects of age in the current study.

\section{Inclusion/Exclusion Criteria}

To participate in this study, potential and willing participants were required to be adults newly diagnosed with HIV (less than 1-month-old clinical diagnosis) registered as receiving antiretroviral therapy at the Adeoyo Maternity Teaching Hospital, must be reporting suicidal ideation because of the new HIV-positive diagnosis and must be able to communicate using English. It was also required that potential participants had no pre-existing illness. The study excluded patients who were not willing to participate, with HIV-positive diagnosis older than a month old, who were not suicidal despite the new HIV-positive diagnosis or who could not communicate using English. The study also excluded patients who were not registered as receiving antiretroviral therapy at the Adeoyo Maternity Teaching Hospital and patients with a pre-existing illness.

\section{Instrument}

A very structured questionnaire was used in this study. It consisted of demographic assessment of participants. Furthermore, it included the scale for suicide ideation by Beck et al. (1979), which is a 19-item clinical instrument that has proven consistent relevance in the clinical assessment of suicidal ideation as a dependent variable in studies in which effectiveness of the treatment was measured (Alavi, et al. 2013). In the current study, the scale for suicide ideation was used to assess the suicide ideation levels of participants (the intervention and control groups) before and after exposure to the treatment. The scoring was performed by adding the singleitem score, and the possible score for participants ranged from 0 to 38 . The authors of the scale reported an internal consistency of 0.89 for the scale, and almost similar reliability coefficient of 0.92 was obtained in this study.

The module of CFT adopted from Gilbert and Procter (2006) and Gilbert (2010) was the instrument that guided the intervention in this study. Adopting this intervention was focused on actively working with the intervention group on compassionate training all through the therapy sessions. In achieving this, therapeutic 
activities that focused on changing self-criticism and suicidal thoughts to compassionate self-correction were performed through means such as self-compassionate quiz, compassionate letter writing and the compassionate thought diaries. The CFT module comprised 10 modules with each module having a specific aim of training. The 10 modules started with a solid building of therapeutic alliance and the introduction of participants to the intervention programme and ended with relapse prevention and group closing.

\section{Procedure}

Permission was sought, and access to the record of patients newly diagnosed with HIV/AIDS receiving antiretroviral therapy was granted for the purpose of determining likely participants. From the record list, a total of 31 new diagnoses were found to have occurred in recent 4 weeks (inclusion criteria); consequently, they were contacted via phone call for invitation to a discussion/ meeting that explained the purpose, procedures and ethical considerations of the study to them. Of the 31 newly diagnosed who were contacted, 28 consented to attend the discussion/ meeting. Before the discussion/meeting commenced, it was quite uneasy getting the attention of likely participants, given the fact that most of them found it uneasy to cope with the new HIV-positive diagnosis. However, after they were all made to understand that the study involved neither risk nor harm and that the study was to help them adjust to the realities of newly living with HIV, they, therefore, willingly expressed interests in the meeting/discussion and the study. After the discussion/ meeting was held, and the newly diagnosed PLWHA consented to participate in the study; they were assessed for suicidal ideation because of the new HIV-positive diagnosis. The families of the newly diagnosed PLWHA and caregivers were also contacted with their consents to establish that their premorbid functioning was not inclusive of suicidality. The results from the assessments showed that of the 28 newly diagnosed PLWHA who attended the meeting/discussion, 22 had thought about suicide after the new HIV-positive diagnosis.

Random assignment was used to equally assign the 22 newly diagnosed PLWHA into two groups. Furthermore, random determination was used to determine which group to be labelled as the intervention or control group. The intervention group received 14 sessions of CFT (2 sessions per week for 7 weeks) in addition to their usual antiretroviral therapy, whereas the control group received only their usual antiretroviral therapy. The researchers maintained contact and relationship with both the intervention and control groups so that there will be no loss of participant throughout the study. The suicidal ideation scale was used to assess suicidal ideation before and after administering CFT, and a comparison of the changes in suicidal ideation was performed between the intervention and control groups before and after the administration of the treatment, using the independent sample t-test on the Statistical Package for Social Sciences (SPSS v. 22.0). To reduce the occurrence of bias and confounding factors, all participants were discouraged from discussing about the events occurring in their group with anyone, and they were blinded from knowing what went on in their counterpart's group. In addition, homework was given to assess the commitments of participants to the intervention programme. Confidentiality of the information was maintained throughout the study, and participants were free to refuse participation at any point of the study with no implication. Both written and verbal consents were also obtained from participants before commencement of the intervention programme. After the intervention study was terminated, the control group was exposed to CFT to enable them benefit from the therapy just as their intervention group counterparts did. Finally, all participants were given incentives, and as a part of the ethical considerations, all participants and the hospital management were assured of feedbacks from the result of the study.

\section{RESULTS}

The demographic information summary presented on table 1 showed that, 5 males and 6 females with a mean age of $39.2 \pm 5.62$, made up the intervention group. It was also observed that, 3 males and 8 females with a mean age of $40.8 \pm 5.67$ made up the control group.

The results presented in Table 2 showed that there was no significant difference in the age of the participants in the intervention and control groups as established by independent sample t-test at $[\mathrm{t}(20)=-0.68, \mathrm{P}=0.91]$. Therefore, this suggested that both groups were similar, and the random assignment was efficacious in minimizing age-related confounding factors amongst participants.

\section{Testing of Hypothesis}

The alternate hypothesis stated that participants exposed to CFT will have significantly reduced suicidal ideation at posttest than at pre-test compared to the participants in the control group, whereas the null hypothesis stated that there will be no significant difference in suicidal ideation between the intervention and control groups before and after exposure to CFT. This was tested using t-test for independent samples, and the results were presented in Table 3. 
Table 1. Demographic Information Summary of Study Participants

\begin{tabular}{|c|c|c|}
\hline Intervention Group & N & $\%$ \\
\hline Male & 5 & 46 \\
\hline Female & 6 & 54 \\
\hline Mean age $=39.2 \pm 5.62$ years & & \\
\hline Control Group & & \\
\hline Male & 3 & 27 \\
\hline Female & 8 & 73 \\
\hline Mean age $=40.8 \pm 5.67$ years & & \\
\hline
\end{tabular}

Table 2. Comparison of Age Differences Between the Intervention and Control Groups to Establish the Confounding Potentiality of Participants' Age

\begin{tabular}{|c|c|c|c|}
\hline $\begin{array}{c}\text { Intervention } \\
\text { Group Mean (SD) }\end{array}$ & $\begin{array}{c}\text { Control } \\
\text { Group Mean (SD) }\end{array}$ & $\mathbf{t}$ (20) & P-value \\
\hline $39.2(5.62)$ & $40.82(5.67)$ & -0.68 & 0.91 \\
\hline
\end{tabular}

Table 3. Independent Sample t-test Summary Table Showing the Changes in Suicidal Ideation Between the Intervention and Control Groups Before and After Exposure to CFT.

\begin{tabular}{|c|c|c|c|c|}
\hline & $\begin{array}{c}\text { Intervention } \\
\text { Group Mean (SD) }\end{array}$ & $\begin{array}{c}\text { Control } \\
\text { Group Mean (SD) }\end{array}$ & $\mathbf{t}(\mathbf{2 0 )}$ & P-Value \\
\hline $\begin{array}{c}\text { Pre- } \\
\text { test }\end{array}$ & $34.91(1.81)$ & $34.82(1.17)$ & 0.14 & 0.17 \\
\hline $\begin{array}{c}\text { Post- } \\
\text { test }\end{array}$ & $0.09(0.30)$ & $35.73(2.20)$ & -53.34 & 0.003 \\
\hline
\end{tabular}

CFT (Independent Variable)

\section{Suicidal Ideation (Dependent Variable)}

The results presented in Table 3 showed that there was no significant difference in the suicidal ideation mean scores of the intervention group (34.91) and the control group (34.82) at the pre-test $[\mathrm{t}(20)=0.14, \mathrm{P}=0.17]$. Interestingly, after exposing participants in the intervention group to CFT, the results showed that there was a significant difference in suicidal ideation, with the intervention group scoring significantly low mean score (0.09) when compared with the control group mean score (35.73) at the post-test $[\mathrm{t}(20)=-53.34, \mathrm{P}=0.003]$. The scores of Cohen's $d$ and power analysis were -0.32 and 0.27 , respectively. Therefore, the alternate hypothesis is retained, whereas the null hypothesis is rejected.

\section{DISCUSSION AND IMPLICATION FOR THE HIV CARE MULTIDISCIPLINARY TEAM}

We carried out an intervention study that determined the efficacy of CFT as an intervention for reducing suicidal ideation in newly diagnosed PLWHA who are presenting suicidal ideation because of the new HIV-positive diagnosis. Our study hypothesised an alternate and a null hypotheses. The results obtained were in line with the stated alternate hypothesis, which led to the acceptance of it. The results demonstrated that the mean scores of the intervention and the control groups were not significantly different at the pre-test; however, at the post-test, it was established that after the exposure to the treatment, the mean score of the intervention group significantly reduced, whereas the mean score of the control group increased because there was no exposure to the treatment for the control group throughout the study. An implication of this result is that suicidal ideation in newly diagnosed PLWHA worsens over time if psychological interventions are not administered early enough.

Although related studies have not established that CFT as an intervention was effective in reducing suicidal risks amongst newly diagnosed PLWHA, the findings in the current study were in line with these related studies that have shown how elements of self-compassion enhances various facets of health and well-being in PLWHA (e.g. O'Donovan \& Gill, 2015; Skinta, et al. 2018; Swansick, 2019; Ironson, et al. 2017). However, findings from the current study disagreed with Webel et al. (2015), who argued that self-compassion does not contribute relevantly to the experience of HIV symptoms amongst PLWHA. In addition, another finding in the present study that showed that suicidal ideation worsens over time if psychological intervention is not administered early enough, was in agreement with the findings of Ongeri et al. (2018), which established that newly diagnosed PLWHA receiving only antiretroviral therapy in a Kenyan general medical facility considered attempting suicide after reporting suicidal ideation in the past month. Therefore, an implication of this suggests that antiretroviral therapy alone is insufficient to address the health needs of PLWHA.

Our current study used a relatively small sample size and obtained a significant result, and this could be possibly supported by previous studies that studied relatively small sample sizes and reported significant results as well, for example, O'Donovan et al. (2015) studied a sample of 10 patients with HIV/AIDS and showed that CFT significantly enhanced adaptive functioning. Therefore, a possible explanation for the finding in our current study, which demonstrated that CFT significantly reduced suicidal ideation, could be found in 
the compassionate mind training contents of the CFT, which postulates that training persons living with shame, self-harm or self-guilt to be compassionate is very imperative for improving their adaptive functioning. This study was, therefore, amongst the first studies to validate the assertions of Gilbert (2014) using a sample of newly diagnosed PLWHA. The findings from this study were also an extension of related Nigerian studies that have tested the effective utility of CFT using vulnerable Nigerian populations. For instance, Agberotimi et al. (2017) determined that CFT was effective in a sample of Nigerian youths with substance use disorders; interestingly, our current study had extended this by establishing that CFT is also effective amongst Nigerians living with HIV and presenting suicidal thoughts.

\section{Recommendation}

Before the initiation of treatment in this study, there was preassessment of study participants, which showed that suicidal thoughts were common amongst newly diagnosed PLWHA because of the new HIV-positive diagnosis. We recommend that HIV pre-testing counselling addressing suicide risks should be an important practice amongst HIV care providers. In addition, PLWHA should be routinely assessed for probable suicidal risks associated with the burden of living with HIV. Second, this study ascertained that CFT was effective in reducing suicidal ideation at the post-assessment of the intervention group; we, therefore, recommend that in addition to antiretroviral medications, CFT should be included in the early treatment plan of HIV-care providers. This will be achieved by encouraging the inclusion of Clinical Health Psychologists into the multidisciplinary team model of HIV care. Third, as revealed by the post-assessment of the control group, it was interestingly established that suicidal ideation increased over time, and this was because of the absence of CFT. In this regard, it is recommended that newly diagnosed PLWHA who are suicidal should receive immediate psychological interventions for the purpose of preventing further suicidal complications in them.

\section{Strength and Weakness of Study}

The strength of this study was the randomisation, and blinding approaches used to limit the chances of biases and confounding factors. However, there could still have been possibilities of other biases that the researchers were unaware of. Another limitation is that this study did not carry out a follow-up study to ascertain the long-term effects of receiving CFT, because of resources beyond the control of the authors. Further studies are, therefore, required to establish the long-term effects of receiving CFT amongst newly diagnosed PLWHA. In addition, the study setting, which is not a special facility for HIV/AIDS treatment, did not give room for accessing a large sample size, and this could have potentially limited the effect size and power analysis results in this study. Therefore, in later studies, a larger sample size could be drawn from multiple HIV/AIDS specialised treatment centres.

\section{CONCLUSION AND FURTHER STUDY}

Our study concluded that CFT has been empirically established as having the potential for reducing suicidal ideation in newly diagnosed PLWHA, and this is a significant addition to knowledge because related studies were not found to have explored the point of focus of the current study. The study also concluded that the absence of early psychological interventions for newly diagnosed PLWHA who are suicidal leads to greater suicidal risks amongst them. It is suggested that further studies should explore the negative biopsychosocial reactions of newly diagnosed PLWHA to a new HIV-positive diagnosis for the purpose of identifying common negative reactions other than suicidal ideation and test for the efficacy of psychological interventions that have not been previously tested.

\section{ACKNOWLEDGEMENT}

We acknowledge our colleagues, Jonathan Kuek, who is a research assistant at the University of Sydney, Australia, and Dr. Samson F. Agberotimi from the North West University, South Africa, for reviewing each phase of the research whilst it was on going. We acknowledge Adenike Oduwusi, MBACP, from the University of Salford, United Kingdom, for her significant inputs on this article and Dr. Omotoso Busayo for aiding our access to the study setting. We also acknowledge all medical personnel and the study participants whose cooperation assisted the research procedures. We acknowledge the Oyo state Ministry of Health for granting ethical approval for this study. We also acknowledge the management of the Adeoyo Maternity Teaching Hospital for the approval they granted us into the hospital.

\section{CONTRIBUTION OF AUTHORS}

First author - Conceptualization, design, intervention program, data collection/analyses, article writing, and proofreading.

Second author - Design, intervention program, data collection/ analyses, article writing, and proofreading.

\section{CONFLICT OF INTEREST}

The authors have no conflict of interest to declare. 


\section{ETHICAL APPROVAL}

Our study was carried out in accordance with the ethical standards of the 1964 Helsinki Declaration. Ethical approval for this study was received from the Ethical Review Board of Oyo state, Nigeria Ministry of Health.

\section{FUNDING}

The study was self-funded.

\section{INFORMED CONSENT}

Verbal and written consents were obtained from all participants in the study.

\section{REFERENCES}

Agberotimi, S. F., Osinowo, H O., Asagba, R. B. Efficacy of compassion-focused therapy in a sample of youths with substance use disorder in Ogbomoso, Nigeria. African Journal of Drug \& Alcohol Studies, 2017; 16(2): 107-116.

Agarwal, A., Mahore, R., Pathak, A. Individual Psychological Reactions to disclosure of HIV positive Diagnosis in Adults victims. The International Journal of Indian Psychology, 2018; Volume 6, Issue 2, DIP:18.01.029/20180602 DOI: 10.25215/0602.029.

Alavi, M. A., Sharifi, B., Ghanizadeh, A., Dehbozorgi, G. Effectiveness of cognitive-behavioral therapy $n$ decreasing suicidal ideation and hopelessness of adolescents with previous suicidal attempts. Iranian Journal of Pediatrics, 2013; 23(4): 467-472.

Avert. HIV and AIDS in Nigeria. 2019; Retrieved from https://www. avert.org/printpdf/node/403

Beck, A. T., Kovacs, M., Weissman, A. Assessment of suicidal ideation: The scale for suicide ideation. Journal of Consulting and Clinical Psychology, 1979; 47(2): 343-352.

Brion, J. M., Leary, M., Drabkin, A. Self-compassion and reactions to serious illness: The case of HIV. Journal of Health Psychology, 2014; 19(2): 218-229.

Carrieri, M. P., Marcellin, F., Fressard, L., Preau, M., SagaonTeyssier, L., Suzan-Monti, M., et al. Suicide risk in a representative sample of people receiving HIV care: Time to target most at-risk populations (ANRSVESPA2 French national survey). PLoS One, 2017; 12(2): e0171645.

Dabaghzadeh, F. Jabbari, F. Khalili, H., Abbasian, L. Associated factors of suicidal thoughts in HIV-positive individuals. Iranian Journal of Psychiatry, 2015; 10(3): 185-191.
Dwyer-Lindgren, L., Cork, M. A., Sligar, A., et al. Mapping HIV prevalence in sub-Saharan Africa between 2000 and 2017. Nature, 2019; 570, 189-193.

Egbe, C. O., Dakum, P. S., Ekong, E., Kohrt, B. A., Minto, J. G., Ticao, C. J. Depression, suicidality, and alcohol use disorder among people living with HIV/AIDS in Nigeria. BMC Public Health. 2017; 17(1): 542. Doi:10.1186/s12889-017-4467-5.

Gebremariam, E. H., Reta, M. M., Nasir, Z., Amdie, F. Z. Prevalence and associated factors of suicidal ideation and attempt among people living with HIV/ADS at Zewitu Memorial Hospital, Addis Ababa, Ethiopia: A cross-sectional study. Psychiatry Journal, 2017; https://doi.org/10.1155/2017/2301524.

Gilbert, P. The CBT distinctive features series. Compassion focused therapy: Distinctive features. Routledge/Taylor \& Francis Group, 2010.

Gilbert, P. The origins and nature of compassion focused therapy. British Journal of Clinical Psychology, 2014; 53(1): https://doi. org/10.1111/bjc.12043.

Gilbert, P., Procter, S. Compassionate mind training for people with high shame and self-criticism: Overview and pilot study of a group therapy approach. Clin. Psychol. Psychother. 2006; 13, 353379.

Harrowing, J. N. Compassion practice by Ugandan nurses who provide HIV care. Online Journal of Issues in Nursing, 2011; 16(1): 5.

Ironson, G., Kremer, H., Lucette, A. Compassionate love predicts long-term survival among people living with HIV followed for up to 17 years. Journal of Positive Psychology, 2017; 1-10. 
Kamangar, F. Confounding variables in epidemiologic studies: Basics and beyond. Archives of Iranian Medicine, 2012; 15(8): 508516.

Kharsany, A. B., Karim, Q. A. (2016). HIV infection and AIDS in subSaharan Africa: Current status, challenges and opportunities. Open AIDS J. 2016; 8, 10-34.

Lingen-Stallard, A., Furber, C., Lavender, T. Testing HIV positive in pregnancy: A phenomenological study of women $\square$ s experiences. Midwifery, 2016; 35, pp. 31-38.

Moradmand-Badie, B., Moayedi-Nia1, S., Foroughi, M., Dejman, M., Nikzad, R., Akhlaghkhah, et al. HIV Positive Patients' Experiences on Receiving an HIV Positive Test: An Iranian - Qualitative Study. American Journal of Epidemiology and Infectious Disease, 2014, Vol. 2, No. 1, 47-51.

Mousa A. Detection and management of confounding variables in prevention of blindness research: A methodological approach with age and sex as models. Austin J. Clin. Ophthalmol, 2014; 1(2): 1006.

NACA Fact Sheet. Fact sheet: HIV prevention program - NACA Nigeria. 2016; Retrieved from hhtps://naca-gov.ng/fact-sheet-hivprevention-program/

Nwobi, U. A., Eseadi, C., Emeka, O., Ekwealor, N., Ogbonnaya, K. A., Oboegbulem, A. I., et al. A stress management intervention for adults living with HIV in Nigerian community setting. Medicine, 2018; 97(47): e13504

O'Donovan, A., Gill, E., Gibson, S. Compassion-focused therapy for people living with HIV: Pilot of a mindfulness and compassionbased cognitive therapy group. HIV Medicine, 2015; 16: 63-63.

Odiachi, A., Erekaha, S, Cornelius, L. J., Isah, C., Ramadhani, H. O., Rapoport, L., Sam-Agudu, N. A. HIV status disclosure to male partners among rural Nigerian women along the prevention of mother-to-child transmission of HIV cascade: A mixed methods study. Reproductive Health, 2018; 15(36): 1-12.

Okoror, T. A., Falade, C. O., Walker, E. M., Olorunlana, A., Anaele, A. Social context surrounding HIV diagnosis and construction of masculinity: A qualitative study of stigma experiences of heterosexual HIV positive men in Southwest Nigeria. BMC Public Health, 2016; 16(507): 1-10.
Oladeji, B. D., Taiwo, B., Mosuro, O., Fayemiwo, S. A., Abiona, T., Fought, A J., et al. Suicidal behavior and associations with quality of life among HIV/AIDS -infected patients in Ibadan, Nigeria. Journal of the International Association of Providers of AIDS Care, 2015; 16(4): 376-382.

Ongeri L., McCulloch, C. E., Neylan, T. C., Bukusi, E., Macfarlane, S. B., Othieno, C., et al. Suicidality and associated risk factors in outpatients attending a general medical facility in rural Kenya. $J$ Affect Disord, 2018; 225: 413-421.

Onyebueke, G. C., Okwaraji, F. E. Depression and suicide risk among HIV positive individuals attending an outpatient HIV/AIDS clinic of a Nigerian tertiary health institution. African Journal of Psychiatry, 2015; 18(1): 1-8.

Ruffieux, Lemsalu, Aebi-Popp, Calmy, Cavassini, Fux, Gunthard, et al. Mortality from suicide among people living with HIV and the general Swiss population: 1988-2017. Journal of the International AIDS Society, 2019; 22(8): 1-8.

Schlebusch, L., Govender, R. D. Elevated risks of suicidal ideation in HIV-positive persons. Depression research and treatment, 2015(4): 1-6.

Skinta, M., Williams, E. M., Fekete, S. L. HIV-stigma, selfcompassion, and psychological wellbeing among gay men living with HIV. Stigma and Health, 2019; 4(2): 179-187.

Swansick, L. "May I be kind to myself": A study on self-compassion and shame on a HIV nightline. Master's project and capstones, 2019; 935, https://repository.usfca.edu/capstone/935.

UNAIDS Data, (2019). Retrieved from https://www.unaids.org/ sites/default/files/media_asset/2019-UNAIDS-data_en.pdf.

Webel, A. R., Wantland, D., Rose, C. D., Kemppainen, J. et al. A cross-sectional relationship between social capital, selfcompassion, and perceived HIV symptoms. Journal of Pain and Symptom Management, 2015; 50(1): 59-68.

Zarei, N., Joulaei, H. The impact of perceived stigma, quality of life, and spiritual beliefs on suicidal ideations among HIV-positive patients. AIDS Research and Treatment, 2018(4); 1-7. 